\title{
INJECTIVITY OF THE PETRI MAP FOR TWISTED BRILL-NOETHER LOCI
}

\author{
MONTSERRAT TEIXIDOR I BIGAS
}

\author{
Abstract. Let $C$ be a generic curve, $E$ a generic vector bundle on $C$. Then, \\ for every line bundle on $C$ the twisted Petri map$$
P_{E}: H^{0}(C, L \otimes E) \otimes H^{0}\left(C, K \otimes L^{*} \otimes E^{*}\right) \rightarrow H^{0}(C, K)
$$ \\ is injective. \\ Mathematical sciences classification number $14 \mathrm{H} 60$.
}

\section{INTRODUCTION}

Let $C$ be an algebraic curve defined over an algebraically closed field. Let $U\left(r_{F}, d_{F}\right)$ be the moduli space of stable vector bundles of rank $r$ and degree $d$. Choose a generic vector bundle $E$ of rank $r_{E}$ and degree $d_{E}$ and denote by $B_{r_{F}, d_{F}}^{k}(E)$ the so called Brill-Noether locus consisting of vector bundles

$$
\left\{F \in U\left(r_{F}, d_{F}\right) \mid \operatorname{dim} H^{0}(C, F \otimes E) \geq k\right\} .
$$

These sets play a crucial role in the geoemetry of moduli spaces of vector bundles. For example, for suitable choices of $E$ and $k=1$ they give the generalized theta divisor providing generators of the Picard group of the moduli space.

Brill-Noether loci can be given scheme structures as locally defined determinantal varieties (see [GT] for an exposition under the additional assumption $E=\mathcal{O}$ ). As such their expected dimension is given by the Brill-Noether number

$\rho=r_{F}^{2}(g-1)+1-k\left(k-r_{F} d_{E}-r_{E} d_{F}+r_{E} r_{F}(g-1)\right)=\operatorname{dim}\left(U\left(r_{F}, d_{F}\right)-h^{0}(F \otimes E) h^{1}(F \otimes E)\right.$.

Here we write $h^{0}(F)$ for the dimension of the space of sections $H^{0}(C, F)$ and the last equality in the formula above assumes that $h^{0}(F \otimes E)=k$. One also expects that when $\rho$ is negative, these loci are empty.

Consider the so-called Petri map

$$
P_{E}(F): H^{0}(C, F \otimes E) \otimes H^{0}\left(C, K \otimes F^{*} \otimes E^{*}\right) \rightarrow H^{0}\left(C, F \otimes K \otimes F^{*}\right)
$$

obtained as the composition of the natural cup-product and tensorization with the identity morphism in $E$. If $h^{0}(F \otimes E)=k$, the tangent space to $B_{r, d}^{k}(E)$ at the point $F$ can be identified to the orthogonal to the image of this map. In particular, $F$ is a non-singular point of a component of dimension $\rho$ of $B_{r, d}^{k}(E)$ if and only if the Petri map is injective. Therefore, proving the injectivity of the Petri map for the generic curve would provide a complete positive answer to the more pressing questions in Brill-Noether Theory. It proves also that when the Brill-Noether number is negative, the locus is actually empty. Moreover, the injectivity of the Petri map helps explain the structure of the tangent cone to the Brill-Noether loci (see [CT]).

In the special case in which $r=1, E=\mathcal{O}$ one recovers the classical Brill-Noether loci in the Jacobian. It is well known that in this particular case all our expectations are satisfied: the loci are non-empty on any curve when $\rho>0$ and the Petri map is injective on the generic curve which implies that these loci have dimension precisely 
$\rho$ and that the singular locus of $B_{r, d}^{k}(E)$ is $B_{r, d}^{k+1}(E)$ (see for instance $\mathrm{ACGH}$ ). On the other hand, when $r>1$, there are counterexamples of particular values of $r, d, k, g$ where the expected results fail, even for the generic curve (see [GT]).

In this paper, we want to deal with the case $r_{F}=1$ but arbitrary $r_{E}$. The nonemptiness result under these hypothesis was proved by Ghione in $G$. Therefore, in order to complete the picture, we need to show the injectivity of the Petri map. We will write $L$ instead of $F$. We can identify $L \otimes K \otimes L^{*}$ with $K$ (as it is done for classical Bril-Noether). We prove the following:

1.1. Theorem Given a generic curve $C$ and a generic vector bundle $E$ on $C$ for any choice of $L \in \operatorname{Pic}^{d}(C)$ on $C$, the Petri map

$$
P_{E}(L): H^{0}(C, L \otimes E) \otimes H^{0}\left(C, K \otimes L^{*} \otimes E^{*}\right) \rightarrow H^{0}(C, K)
$$

is injective. In particular, the twisted Brill-Noether locus $B_{1, d}^{k}(E)$ is of the expected dimension $\rho$ and singular only along $B_{1, d}^{k+1}(E)$

In order to prove the injectivity of the Petri map for a generic curve, it suffices to prove it for a special curve. We'll choose our curve to be reducible with components rational and elliptic. Vector bundles on these curves are easy to describe in terms of the restrictions of the vector bundles to the various components and gluing at the nodes. We choose a generic such $E$ and by means of limit linear series prove the result. The tools used are those developed in T3 which in turn generalizes EH2.

\section{Preliminaries on Reducible curves}

Consider a family of curves $\pi: \mathcal{C} \rightarrow T$. Let $T$ be the spectrum of a discrete valuation ring $\mathcal{O}$ with maximal ideal generated by $t$. Assume that the generic fiber of $\pi$ is a non-singular curve and the special fiber $C$ looks as follows:

Take $g$ elliptic curves $C^{i}, i=1, \ldots g$ and let $P^{i}, Q^{i}$ be generic points on $E^{i}$. Take any number of rational curves $C_{1}^{0}, . . C_{k_{0}}^{0}, \ldots C_{1}^{g} \ldots C_{k_{g}}^{g}$ again with points $P_{j}^{i}, Q_{j}^{i}$ on them. Glue $C_{j}^{i}$ to $C_{j+1}^{i}$ by identifying $Q_{j}^{i}$ to $P_{j+1}^{i}$. Glue $C_{k_{i-1}}^{i-1}$ to $C^{i}$ by identifying $Q_{k_{i-1}}^{i-1}$ to $P^{i}$. Glue $C^{i}$ to $C_{1}^{i}$ by identifying $Q^{i}$ to $P_{1}^{i}$.

For convenience of notation, we shall denote by

$$
Y_{1}, \ldots Y_{M}, M=k_{0}+\ldots+k_{g}+g
$$

the components of $C$ starting with $C_{1}^{0}$ and ending with $C_{k_{g}}^{g}$. We shall denote by $P_{i}, Q_{i}$ the two points in $Y_{i}$ that get identified to $Q_{i-1} \in Y_{i-1}$ and $P_{i+1} \in$ $Y_{i+1}$ respectively. We will use superindices when we need to refer to the elliptic components, so $C^{i}$ will be the $i^{\text {th }}$ elliptic curve..

Note that the form of the central fiber does not change if we make base changes and normalizations.

Given a vector bundle $E$ and a line bundle $L$ on the generic fiber, we can assume that we have extensions $\mathcal{E}, \mathcal{L}$ to the whole family.

If we tensor $\mathcal{E} \otimes \mathcal{L}$ with a line bundle of the form $\mathcal{O}_{\mathcal{C}}\left(\sum \lambda_{i} Y_{i}\right)$, the restriction of the vector bundle to the central fiber does not change while the restriction to special fibers changes its degree in multiples of $r$. In this way,we can concentrate most of the degree and therefore all of the sections on one component of our choice. This is the idea behind the definition of limit linear series (see [EH1, [T3]) 
2.1. Definition. Limit linear series A limit linear series of rank $r$, degree $d$ and dimension $k$ on a chain of $M$ (not necessarily rational and elliptic) curves consists of data I,II below for which data III, IV exist satisfying conditions a-c.

I) For every component $Y_{i}$, a vector bundle $E_{i}$ of rank $r$ and degree $d$ and a $k$-dimensional space of sections $V_{i}$ of $E_{i}$.

II) For every node obtained by gluing $Q_{i}$ and $P_{i+1}$ an isomorphism of the projectivisation of the fibers $\left(E_{i}\right)_{Q_{i}}$ and $\left(E_{i+1}\right)_{P_{i+1}}$

III) A positive integer a

$I V)$ For every node obtained by gluing $Q_{i}$ and $P_{i+1}$, bases $s_{Q_{i}}^{t}, s_{P_{i+1}}^{t}, t=1 \ldots k$ of the vector spaces $V_{i}$ and $V_{i+1}$

Subject to the conditions

a) $\sum_{i=1}^{M} d_{i}-r(M-1) a=d$

b) The orders of vanishing at $P_{i+1}, Q_{i}$ of the sections of the chosen basis satisfy $\operatorname{ord}_{P_{i+1}} s_{i+1}^{t}+\operatorname{ord}_{Q_{i}} s_{i}^{t} \geq a$

c) Sections of the vector bundles $E_{i}\left(-a P_{i}\right), E_{i}\left(-a Q_{i}\right)$ are completely determined by their value at the nodes.

Note that up to replacing $E$ by $E \otimes L_{0}$ and $L$ by $L \otimes L_{0}^{-1}$, we can assume that $L$ has degree zero, so this will be understood in what follows. We then have to deal with only one rank and degree, so we will drop the subindex $E$ and write $r, d$ for the degree and rank of $E$.

Write $d=r d_{1}+d_{2}, 0 \leq d_{2}<r$. Denote by $h$ the greatest common divisor of $r, d$ and write $r=h r^{\prime}, d_{2}=h d_{2}^{\prime}$.

In order to prove the result for a generic $E$, it suffices to prove it for a particular $E$. We take as vector bundle on the special curve one with restriction to the first $g-1$ elliptic components and all of the rational components a direct sum of line bundles of the same degree while the restriction to the last elliptic component is a direct sum of $h$ stable vector bundle of same rank and degree.

Choose a component $Y_{i}$. Modifying the vector bundle on the central fiber by tensoring with line bundles of the form $\mathcal{O}_{\mathcal{C}}\left(\sum \lambda_{i} Y_{i}\right)$, we obtain a vector bundle $\mathcal{E}_{i}$ such that

$$
\begin{gathered}
Y_{i} \neq C^{g}, \operatorname{deg}\left(\mathcal{E}_{i \mid Y_{i}}\right)=r d_{1}, \operatorname{deg}\left(\mathcal{E}_{i \mid Y_{j}}\right)=0, Y_{j} \neq Y_{i}, Y_{j} \neq C^{g}, \operatorname{deg}\left(\mathcal{E}_{i \mid C^{g}}\right)=d_{2} \\
Y_{i}=C^{g}, \operatorname{deg}\left(\mathcal{E}_{i \mid Y_{i}}\right)=d, \operatorname{deg}\left(\mathcal{E}_{i \mid Y_{j}}\right)=0, Y_{j} \neq Y_{i}
\end{gathered}
$$

Then, the $E_{i}$ that appears in II of 2.1 is $\mathcal{E}_{i \mid Y_{i}}$. The $a$ of III in 2.1 is $a=d_{1}$.

We do the same procedure with $K \otimes E^{*} \otimes L^{*}$. In this case, write $\mathcal{E}^{\prime}$ for $\omega \otimes \mathcal{E}^{*} \otimes \mathcal{L}^{*}$. Then,

$$
\begin{gathered}
Y_{i} \neq C^{g}, \operatorname{deg}\left(\mathcal{E}_{i \mid Y_{i}}^{\prime}\right)=r\left(2 g-2-d_{1}\right), \operatorname{deg}\left(\mathcal{E}_{i \mid Y_{j}}^{\prime}\right)=0, Y_{j} \neq Y_{i}, Y_{j} \neq C^{g}, \operatorname{deg}\left(\mathcal{E}_{i \mid C^{g}}\right)=-d_{2} \\
Y_{i}=C^{g}, \operatorname{deg}\left(\mathcal{E}_{i \mid Y_{i}}^{\prime}\right)=r(2 g-2)-d, \operatorname{deg}\left(\mathcal{E}_{i \mid Y_{j}}^{\prime}\right)=0, Y_{j} \neq Y_{i}
\end{gathered}
$$

Then, $a^{\prime}=2 g-3-d_{1}$.

2.2. Lemma The canonical limit linear series on $C$ has line bundles on $C^{i}$ equal to $K^{i}=\mathcal{O}\left(2(i-1) P^{i}+2(g-i) Q^{i}\right)$ while on the rational components the line bundle is $\mathcal{O}(2(g-1))$. The space of sections on $C^{i}$ is $H^{0}\left(L_{i}(-(i-2) P-(2 g-2 i) Q)\right) \oplus$ $H^{0}\left(L_{i}(-(2 i-1) P-(g-i-1) Q)\right)$. The unique section whose order of vanishing at $P$ and $Q$ is $2 g-2$ vanishes with order $2(i-1)$ at $P$ and $2 g-2 i$ at $Q$. 


\section{VANISHING AT THE NODES OF ELEMENTS IN THE KERNEL}

Let us assume that the twisted Petri map is not injective on the generic curve. Then we can find sections in the kernel of the Petri maps

$$
\pi_{*}(\mathcal{E} \otimes \mathcal{L})_{i} \otimes \pi_{*}\left(\omega_{\pi} \otimes \mathcal{E}^{*} \otimes \mathcal{L}^{*}\right)_{i} \rightarrow \pi_{*} \omega_{\pi i}
$$

that glue with each other as we go from one component to the next. We next study the vanishing of these sections at the various nodes.

3.1. Lemma. (see [EH2] 1.2 and [T3] 4.1). For every component $Y_{i}$, there is a basis $\sigma_{j}, j=1 \ldots k$ of $\pi_{*} \mathcal{E}_{i}$ such that

a) $\operatorname{ord}_{P_{i}}\left(\sigma_{j}\right)=a_{j}\left(P_{i}\right)$

b) for suitable integers $\alpha_{j}, t^{\alpha_{j}} \sigma_{j}$ are a basis of $\pi_{*}\left(\mathcal{E}_{i+1}\right)$

3.2. Proposition (see[T3] 4.2) Consider a vector bundle $\mathcal{F}$ on $\pi: \mathcal{C} \rightarrow T$ as before associated to a vector bundles $F$. Let $\sigma_{j}$ be a basis of $\pi_{*} \mathcal{F}_{i}$ such that $t^{\alpha_{j}} \sigma_{j}$ is a basis of $\pi_{*}\left(\mathcal{F}_{i+1}\right)$. Let $\bar{a}$ be as in II of 2.1. Then, the orders of vanishing of the $\sigma_{j}$ at the nodes satisfy

a) $\operatorname{ord}_{P_{i}}\left(\sigma_{j}\right) \leq \bar{a}-\operatorname{ord}_{Q_{i}} \sigma_{j} \leq \alpha_{j} \leq \operatorname{ord}_{P_{i+1}} t^{\alpha_{j}} \sigma_{j}$ if $F_{i}$ is a direct sum of $h$ indecomposable vector bundle of rank $r^{\prime}$ and degree $r^{\prime} \bar{a}+\bar{d}_{2}, 0<\bar{d}_{2}<r^{\prime}$.

b) $\operatorname{ord}_{P_{i}}\left(\sigma_{j}\right) \leq \bar{a}-\operatorname{ord}_{Q_{i}} \sigma_{j} \leq \alpha_{j}-1 \leq \operatorname{ord}_{P_{i+1}} t^{\alpha_{j}} \sigma_{j}-1$ if $F_{i}$ is a direct sum of $h$ indecomposable vector bundle of rank $r^{\prime}$ and degree $r^{\prime}(\bar{a}-1)+\bar{d}_{2}, 0<\bar{d}_{2}<r^{\prime}$.

c) $\operatorname{ord}_{P_{i}}\left(\sigma_{j}\right) \leq \bar{a}-\operatorname{ord}_{Q_{i}} \sigma_{j} \leq \alpha_{j} \leq \operatorname{ord}_{P_{i+1}} t^{\alpha_{j}} \sigma_{j}$ if $F_{i}$ is a direct sum of line bundles of degree $\bar{a}$.

Moreover, if equality holds, then $\sigma_{j}$ as a section of $F_{i}$ vanishes at $P_{i}, Q_{i}$ with orders adding up to $\bar{a}$ in a), c) and $\bar{a}-1$ in $b$ ).

Consider now the Petri map

$$
\pi_{*}(\mathcal{L} \otimes \mathcal{E}) \otimes \pi_{*}\left(\omega_{\pi} \otimes \mathcal{L}^{-1} \otimes \mathcal{E}^{*}\right) \rightarrow \pi_{*} \omega_{\pi}
$$

As in $\left[\mathrm{EH} 2\right.$, p.277, one can define the order of a section $\rho \in \pi_{*} \mathcal{L} \otimes \mathcal{E}_{Y} \otimes \pi_{*}\left(\omega_{\pi} \otimes\right.$ $\left.\mathcal{L}^{-1} \otimes \mathcal{E}^{*}\right)$ at a point $P$ on a component $Y$ as follows:

3.3. Definition $W e$ say $\operatorname{ord}_{P}\left(\rho_{\mid Y}\right) \geq l$ if and only if $\rho$ is in the linear span of $t\left(\pi_{*} \mathcal{L} \otimes \mathcal{E}_{Y} \otimes \pi_{*}\left(\omega_{\pi} \otimes \mathcal{L}^{-1} \otimes \mathcal{E}^{*}\right)\right)$ and elements of the form $\sigma_{m} \otimes \sigma_{n}^{\prime}$ where ord ${ }_{P}\left(\sigma_{m}\right)+$ $\operatorname{ord}_{P}\left(\sigma_{n}^{\prime}\right) \geq l, \sigma_{m} \in \pi_{*}\left(\mathcal{L} \otimes \mathcal{E}_{Y}\right), \sigma_{n}^{\prime} \in \pi_{*}\left(\omega_{\pi} \otimes \mathcal{L}^{-1} \otimes \mathcal{E}^{*}\right)$.

One then has the following result (cf. [EH2], Lemma 3.2)

3.4. Lemma Let $\sigma_{m}$ be a basis of the free $\mathcal{O}$ module $\pi_{*}(\mathcal{L} \otimes \mathcal{E})_{i}$ such that the orders of vanishing of the $\sigma_{m}$ at $P_{i}$ are the orders of vanishing of the linear series at this point and $t^{\alpha_{m}} \sigma_{m}$ is a basis of $\pi_{*}(\mathcal{L} \otimes \mathcal{E})_{i+1}$. Let $\sigma_{n}^{\prime}$ be a basis of the free $\mathcal{O}$ module $\left.\pi_{*}\left(\omega_{\pi} \otimes \mathcal{L}^{-1} \otimes \mathcal{E}^{*}\right)\right)$ such that the orders of vanishing of the $\sigma_{n}^{\prime}$ at $P_{i}$ are the distinct orders of vanishing of the linear series at this point and $t^{\alpha_{n}^{\prime}} \sigma_{n}^{\prime}$ is a basis of $\left.\pi_{*}\left(\left(\omega_{\pi} \otimes \mathcal{L}^{-1} \otimes \mathcal{E}^{*}\right)\right)_{i+1}\right)$. If

$$
\rho=\sum f_{n, m}\left(\sigma_{m} \otimes \sigma_{n}^{\prime}\right)
$$

where the $f_{n, m}$ are functions on the discrete valuation ring $\mathcal{O}$ and the associated discrete valuation is $\nu$, then

$$
\operatorname{ord}_{P_{i}}\left(\rho_{\mid Y_{i}}\right)=\min _{\left\{\nu\left(f_{n, m}\right)=0\right\}}\left(\operatorname{ord}_{P_{i}}\left(\sigma_{m}\right)+\operatorname{ord}_{P_{i}}\left(\sigma_{n}^{\prime}\right)\right)
$$

If $\beta_{i}$ is the unique integer such that

$$
t^{\beta_{i}} \rho \in \pi_{*}\left(\mathcal{L}_{i} \otimes \mathcal{E}\right)_{i} \otimes \pi_{*}\left(\omega_{\pi} \otimes \mathcal{L}^{-1} \otimes \mathcal{E}^{*}\right)_{i}-t\left(\pi_{*} \mathcal{L} \otimes \mathcal{E}_{i} \otimes \pi_{*}\left(\omega_{\pi} \otimes \mathcal{L}^{-1} \otimes \mathcal{E}^{*}\right)_{i}\right)
$$


then

$$
\beta_{i}=\max \left\{\alpha_{m}+\alpha_{n}^{\prime}-\nu\left(f_{n m}\right)\right\}
$$

Let us assume now that the kernel of the Petri map is non-zero on the generic curve. We can then find an element $\rho$ such that say

$$
t^{\beta_{i}} \rho \in \pi_{*}(\mathcal{L} \otimes \mathcal{E})_{i} \otimes \pi_{*}\left(\omega_{\pi} \otimes \mathcal{L}^{-1} \otimes \mathcal{E}^{*}\right)_{i}-t\left(\pi_{*}(\mathcal{L} \otimes \mathcal{E})_{i} \otimes \pi_{*}\left(\omega_{\pi} \otimes \mathcal{L}^{-1} \otimes \mathcal{E}^{*}\right)_{i}\right)
$$

and $t^{\beta_{i+1}} \rho \in \pi_{*}(\mathcal{L} \otimes \mathcal{E})_{i+1} \otimes \pi_{*}\left(\omega_{\pi} \otimes \mathcal{L}^{-1} \otimes \mathcal{E}^{*}\right)_{i+1}$ and in the kernel of the twisted Petri map to $\pi_{*}\left(\omega_{\pi}\right)_{i+1}$.

\subsection{Proposition}

1) If $Y_{i}$ is any component,

$$
\operatorname{ord}_{P_{i+1}}\left(t^{\beta_{i+1}} \rho_{\mid Y_{i+1}}\right) \geq \operatorname{ord}_{P_{i}}\left(t^{\beta_{i}} \rho_{\mid Y_{i}}\right)
$$

2) If $Y_{i}$ is an elliptic component, $Y_{i} \neq C^{g}$

$$
\operatorname{ord}_{P_{i+1}}\left(t^{\beta_{i+1}} \rho_{\mid Y_{i+1}}\right) \geq \operatorname{ord}_{P_{i}}\left(t^{\beta_{i}} \rho_{\mid Y_{i}}\right)+1
$$

If $Y_{i}$ is the first component for which there is equality in the above inequality, then the terms of $\rho_{i}$ that give the vanishing at $Q_{i}$ can be written in the form

$$
(*) \bar{\sigma} \otimes \sigma^{\prime}+\sigma \otimes \bar{\sigma}^{\prime}
$$

for two specific sections $\bar{\sigma} \in(\mathcal{L} \otimes \mathcal{E})_{i}$ and $\bar{\sigma}^{\prime} \in \pi_{*}\left(\omega_{\pi} \otimes \mathcal{L}^{-1} \otimes \mathcal{E}^{*}\right)_{i}$. Moreover, the fibers of $\sigma, \sigma^{\prime}$ at the nodes move in spaces of dimension at most $r-1$ complementary to the directions of $\bar{\sigma}, \bar{\sigma}^{\prime}$.

If $\rho_{i-1}$ is of the form (*), then

$$
\operatorname{ord}_{P_{i+1}}\left(t^{\beta_{i+1}} \rho_{\mid Y_{i+1}}\right) \geq \operatorname{ord}_{P_{i}}\left(t^{\beta_{i}} \rho_{\mid Y_{i}}\right)+2
$$

and if there is equality in this inequality, then (*) holds again in the component $Y_{i}$..

3) If $Y_{i}$ is the last elliptic component, $Y_{i}=C^{g}$

$$
\operatorname{ord}_{P_{i+1}}\left(t^{\beta_{i+1}} \rho_{\mid Y_{i+1}}\right) \geq \operatorname{ord}_{P_{i}}\left(t^{\beta_{i}} \rho_{\mid Y_{i}}\right)+1
$$

If $\rho_{i-1}$ is of the form $\left({ }^{*}\right)$, then the inequality above is strict.

Proof. We first prove that 1) holds on any component. Choose a basis $\sigma_{m}, m=$ $1 \ldots k$ of $\pi_{*}\left(\mathcal{L} \otimes \mathcal{E}_{i}\right)$ such that $t^{\alpha_{m}} \sigma_{m}$ is a basis of $\pi_{*}(\mathcal{L} \otimes \mathcal{E})_{i+1}$. Similarly, choose a basis $\sigma_{n}^{\prime}, n=1 \ldots k^{\prime}=k-r d+r(g-1)$ of $\pi_{*}\left(\omega_{\pi} \otimes \mathcal{L}^{-1} \otimes \mathcal{E}\right)_{i}$ such that $t^{\alpha_{n}^{\prime}} \sigma_{n}^{\prime}$ is a basis of $\pi_{*}\left(\omega_{\pi} \otimes \mathcal{L}^{-1} \otimes \mathcal{E}_{i+1}^{*}\right)$. For simplicity of notation, we shall assume that $\beta_{i}=0$. Write

$$
\rho=\sum_{m, n} f_{m, n}\left(\sigma_{m} \otimes \sigma_{n}^{\prime}\right)
$$

Then, from 3.4 .

$$
\operatorname{ord}_{P_{i}}\left(\rho_{\mid Y_{i}}\right)=\min _{\left\{\nu\left(f_{m, n}\right)=0\right\}}\left(\operatorname{ord}_{P_{i}}\left(\sigma_{m}\right)+\operatorname{ord}_{P_{i}}\left(\sigma_{n}^{\prime}\right)\right)
$$

Assume that this minimum is attained by a pair corresponding to the indices $m_{0}, n_{0}$ with $\nu\left(f_{m_{0}, n_{0}}\right)=0$. Then from 3.2 .

$$
\left(\operatorname{ord}_{P_{i}}\left(\sigma_{m_{0}}\right)+\operatorname{ord}_{P_{i}}\left(\sigma_{n_{0}}^{\prime}\right)\right) \leq a+a^{\prime}-\operatorname{ord}_{Q_{i}}\left(\sigma_{m_{0}}\right)-\operatorname{ord}_{Q_{i}}\left(\sigma_{n_{0}}^{\prime}\right) \leq \alpha_{m_{0}}+\alpha_{n_{0}}^{\prime}
$$

From 3.4 and the fact that $\nu\left(f_{n_{0}, m_{0}}\right)=0$, the latter is at most $\beta_{i+1}$.

Write

$$
t^{\beta_{i+1}} \rho=\sum_{n \leq m}\left(t^{\beta_{i+1}-\alpha_{m}-\alpha_{n}^{\prime}} f_{n m}\right)\left(t^{\alpha_{m}} \sigma_{m} \otimes t^{\alpha_{n}^{\prime}} \sigma_{n}^{\prime}\right)
$$


Hence, from 3.4

$\operatorname{ord}_{P_{i+1}}\left(t^{\beta_{i+1}} \rho_{\mid Y_{i+1}}\right)=\min _{\left\{\beta_{i+1}-\alpha_{m}-\alpha_{n}^{\prime}+\nu\left(f_{n m}\right)=0\right\}}\left(\operatorname{ord}_{P_{i+1}}\left(t^{\alpha_{m}} \sigma_{m}\right)+\operatorname{ord}_{P_{i+1}}\left(t^{\alpha_{n}} \sigma_{n}\right)\right)$

Assume that this minimum is attained at a pair $m_{1}, n_{1}$ with

$$
\beta_{i+1}-\alpha_{m_{1}}-\alpha_{n_{1}}^{\prime}+\nu\left(f_{m_{1}, n_{1}}\right)=0
$$

Then,

$$
\left.\beta_{i+1} \leq \beta_{i+1}+\nu\left(f_{m_{1}, n_{1}}\right)=\alpha_{m_{1}}+\alpha_{n_{1}}^{\prime} \leq \operatorname{ord}_{P_{i+1}}\left(t^{\alpha_{m_{1}}} \sigma_{m_{1}}\right)+\operatorname{ord}_{P_{i+1}}\left(t^{\alpha_{n_{1}}^{\prime}} \sigma_{n_{1}}^{\prime}\right)\right)
$$

where the last inequality comes from 3.2

Stringing together the above inequalities, we obtain

$$
\operatorname{ord}_{P_{i}}\left(\rho_{\mid Y_{i}}\right) \leq \operatorname{ord}_{P_{i+1}}\left(\rho_{\mid Y_{i+1}}\right) \text {. }
$$

Hence part 1) is proved.

Assume now that there is equality in the inequality above and we are in the situation of $\mathrm{b}$ ). Then all the previous inequalities must be equalities. In particular, any terms $\sigma_{m} \otimes \sigma_{n}^{\prime}$ that give the vanishing of $\rho$ at $P_{i}$ satisfy

$$
\operatorname{ord}_{P_{i}}\left(\sigma_{m}\right)+\operatorname{ord}_{Q_{i}}\left(\sigma_{m}\right)=a, \operatorname{ord}_{P_{i}}\left(\sigma_{n}^{\prime}\right)+\operatorname{ord}_{Q_{i}}\left(\sigma_{n}^{\prime}\right)=a^{\prime} .
$$

Write $\mathcal{E}_{i}$ as a direct sum of $r$ line bundles of degree $d_{1}$

$$
\mathcal{E}_{i}=L_{1}^{i} \oplus \ldots \oplus L_{r}^{i}
$$

By the genericity of $E^{\prime}$, the $L_{j}^{i}$ are generic. Then,

$$
(\mathcal{E} \otimes \mathcal{L})_{i}=\left(L_{1}^{i} \otimes L^{i}\right) \oplus \ldots \oplus\left(L_{r}^{i} \otimes L^{i}\right) .
$$

and at most one of the $L_{j}^{i} \otimes L^{i}$ is of the form $\mathcal{O}\left(a_{j}^{i} P_{i}+\left(d_{1}-a_{j}^{i}\right) Q_{i}\right)$. Denote this index (if it exists) by $j_{i}$. On an elliptic curve, with $P_{i}, Q_{i}$ generic points, there is only one section $\bar{\sigma}^{i}$ of a line bundle of degree $d_{1}$ with orders of vanishing at $P_{i}, Q_{i}$ adding up to $d_{1}$, the one corresponding to $a_{j}^{i} P_{i}+\left(d_{1}-a_{j}^{i}\right) Q_{i}$. Hence, there is only one section of $(\mathcal{E} \otimes \mathcal{L})_{i}$ with orders of vanishing at $P_{i}, Q_{i}$ adding up to $d_{1}$, the one corresponding to $a_{j}^{i} P_{i}+\left(d_{1}-a_{j}^{i}\right) Q_{i}$ on the $j_{i}^{t h}$ component and zeros elsewhere.

From the description of the limit canonical series, at most one of the $K \otimes L_{j}^{i *} \otimes L^{i *}$ is of the form $\mathcal{O}\left(a_{j}^{\prime i} P_{i}+\left(a^{\prime}-a_{j}^{i}\right) Q_{i}\right)$ and it corresponds to the same $j_{i}$ as before. Therefore, there is only one section $\bar{\sigma}^{i}$ of $\left(\omega_{\pi} \otimes \mathcal{E}^{*} \otimes \mathcal{L}^{*}\right)_{i}$ with orders of vanishing at $P_{i}, Q_{i}$ adding up to $2 g-2-d_{1}$ and again the only non-zero part of this section corresponds to the summand $j_{i}$ in the direct sum decomposition of the vector bundle.

As $\bar{\sigma}^{i} \otimes \bar{\sigma}^{i}$ is not in the kernel of the Petri map, the vanishing must go up by at least one and if it goes up by exactly one, the section $\rho$ is of the form given in $\left.{ }^{*}\right)$. Moreover, the $j_{i}^{t h}$ component of $\bar{\sigma}^{i}, \bar{\sigma}^{\prime i}$ is a section of the canonical bundle that vanishes only at $P_{i}, Q_{i}$. It follows from 2.2 that it vanishes at $P_{i}$ to order $2(i-1)$.

Assume now that this $Y_{i}$ is the first elliptic component $C^{m}$ where the vanishing goes up by just one. Then,

$$
\operatorname{ord}_{P_{i}}(\rho) \geq 2 m-2 .
$$

Write $\sigma=\left(\sigma_{1}, \ldots, \sigma_{r}\right), \sigma^{\prime}=\left(\sigma_{1}^{\prime}, \ldots \sigma_{r}^{\prime}\right)$ according to the decomposition of $\mathcal{E}_{i}$. We want to prove that if the vanishing goes up by exactly one, then $\sigma_{j_{i}}=0, \sigma_{j_{i}}^{\prime}=0$. This will show that the fibers of $\sigma, \sigma^{\prime}$ at the nodes move in spaces of dimension at most $r-1$ complementary to the directions of $\bar{\sigma}, \bar{\sigma}^{\prime}$.

By assumption, $\operatorname{ord}_{P_{i}}(\rho)=\operatorname{ord}_{P_{i}}\left(\bar{\sigma}^{i}\right)+\operatorname{ord}_{P_{i}}\left(\sigma^{\prime}\right)=\operatorname{ord}_{P_{i}}(\sigma)+\operatorname{ord}_{P_{i}}\left(\bar{\sigma}^{\prime i}\right)$. Assume that the $j_{i}$ component of $\sigma^{\prime}$ is non-zero. Then, both this and the $j_{i}$ component of $\bar{\sigma}^{\prime}$ are sections of $\left(L_{j_{i}}^{i} \otimes L^{i}\right)^{\prime}$. Hence, they vanish at different orders at $P_{i}$. 
Then

$2 m-2 \leq \operatorname{ord}_{P_{i}}(\rho)=\operatorname{ord}_{P_{i}}\left(\bar{\sigma}^{i}\right)+\operatorname{ord}_{P_{i}}\left(\sigma^{\prime}\right) \neq \operatorname{ord}_{P_{i}}\left(\bar{\sigma}^{i}\right)+\operatorname{ord}_{P_{i}}\left(\bar{\sigma}^{i}\right)=2 m-2$.

Hence, $\operatorname{ord}_{P_{i}}\left(\bar{\sigma}^{i}\right)+\operatorname{ord}_{P_{i}}\left(\sigma^{\prime}\right) \geq 2 m-1$.

From 3.2 .

$$
\operatorname{ord}_{P_{i}}\left(\sigma^{\prime}\right) \leq 2 g-2-d-\operatorname{ord}_{Q_{i}}\left(\sigma^{\prime}\right) \leq \alpha^{\prime} \leq \operatorname{ord}_{P_{i+1}}\left(t^{\alpha} \sigma^{\prime}\right) .
$$

The first inequality is strict. Hence,

$$
\begin{aligned}
& \operatorname{ord}_{P_{i+1}}\left(t^{\alpha_{i}} \rho\right)=\operatorname{ord}_{P_{i+1}}\left(t^{\bar{\alpha}} \bar{\sigma}^{i}\right)+\operatorname{ord}_{P_{i+1}}\left(t^{\alpha^{\prime}} \sigma^{\prime}\right) \geq \\
& \operatorname{ord}_{P_{i}}\left(\bar{\sigma}^{i}\right)+\operatorname{ord}_{P_{i}}\left(\sigma^{\prime}\right)+1 \geq 2 m-1+1=2 m .
\end{aligned}
$$

If $\rho_{i-1}$ is of the form $(*)$, the genericity of the gluing at the node between the components $Y_{i-1}$ and $Y_{i}$ means that two sections of the form $\left(^{*}\right)$ corresponding to the sections with highest vanishing in each component will not glue together. Therefore, the vanishing in the next component increases in at least two.

Assume now that it increases in precisely two units. By the genericity of the gluing, none of the the sections that glue with each of $\bar{\sigma}^{i-1}, \sigma_{i-1}^{\prime}, \sigma^{i-1}, \bar{\sigma}^{\prime i-1}$ can be $\bar{\sigma}^{i}, \bar{\sigma}^{\prime}$. Therefore, for each of them the sum of vanishing at the two nodes must be one less than the maximum, namely $a-1$. As $E^{i}$ is a direct sum of line bundles of degree $a$, there is a unique section up to scalars whose order of vanishing at the two nodes is $a-1$ and glues with a preassigned direction at $P_{i}$. Hence, the fiber at $Q_{i}$ of $\rho$ is completely determined and the last statement of 2) is proved.

Consider now the last elliptic component. Then, the sum of the orders of vanishing between $P_{i}, Q_{i}$ of a section of $\mathcal{E}_{i}$ is at most $a$ and there is a space of dimension $d_{2}$ of such sections. The sum of the orders of vanishing between $P_{i}, Q_{i}$ of a section of $\mathcal{E}^{\prime}{ }_{i}$ is at most $a^{\prime}-1$ and there is a space of dimension $r-d_{2}$ of such sections. From the arguments above, this implies that the vanishing increases in at least one. Moreover, if it increases in exactly one, then $\rho_{i}$ can be written in the form $\sum \sigma_{k} \otimes \sigma_{k}^{\prime}$ where all the $\sigma_{k}, \sigma_{k}^{\prime}$ are in the special directions mentioned above. In particular, this $\rho$ does not glue with one of the form in $(*)$. Hence, 3$)$ is proved.

The proof of the Theorem now follows from the previous proposition : we have shown that on rational components the vanishing at the nodes does not decrease while on elliptic components it increases in at least one and in all but one component it increases in at least two. This implies that the vanishing at one point in the last component is at least $2 g-1$. This is impossible, as the canonical line bundle has degree $2 g-2$.

\section{REFERENCES}

[A] M.Atiyah, Vector bundles over an elliptic curve Proc. London Math.Soc.(3),7 (1957, 414452.

[ACGH] E.Arbarello, M.Cornalba, P. Griffiths, J.Harris Geometry of algebraic curves, Grundl.math.Wiss. 267 1984, 386pp.

[CT] S.Casalaina-Martin, M.Teixidor Singularities of Brill-Noether loci for vector bundles on a curve arxiv 07102480

[EH1] D.Eisenbud, J.Harris, Limit linear series, basic theory, Invent.Math. ? (1986), 337-371.

[EH2] D.Eisenbud, J.Harris, A simpler proof of the Gieseker-Petri Theorem on special divisors, Invent.Math. 74 (1983), 269-280.

[G] F.Ghione, Un probléme du type Bril-Noether pour les fibrés vectorielsIn Algebraic Geometry-open problems, LNM 997 1983, 197-209.

[GT] I. Grzegorczyk, M.Teixidor, Brill-Noether Theory for stable vector bundles arXiv:0801.4740

[T1] M.Teixidor, Brill-Noether Theory for stable vector bundles Duke Math J62 N2(1991), 385400. 
[T2] M.Teixidor, Moduli spaces of semistable vector bundles on tree-like curves, Math Ann. 290 (1991), 341-348.

[T3] M.Teixidor, Petri map for rank two bundles with canonical determinant. Comp. Math. 144 no. 3 (2008), 705-720.

Mathematics Department, Tufts University, Medford MA 02155

E-mail address: montserrat.teixidoribigas@@tufts.edu 